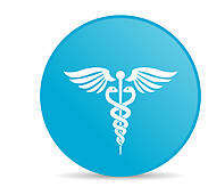

International Journal of Advances in Pharmacy and Biotechnology

Vol.1, Issue-4, 2015, 1-6

Review Article

Open Access.

I J A P B

ISSN: $2454-8375$

\title{
A REVIEW ON DRY POWDER INHALERS
}

\section{Prasanna Kumar Desu, Mamatha Bellamkonda}

Department of Pharmaceutics, St. Mary’s Group of Institutions Guntur, Narakoduru, Guntur, AP, India.

*Corresponding author e-mail: prasanna.desu@gmail.com

\section{ABSTRACT:}

A Dry powder inhaler (DPI) is a dry-powder product that provides medicine to the lungs. Asthma, bronchitis nd COPD are common in treating breathing disorders. DPI interests have been more active in recent years as an effective and environmentally sustainable way to supply medicines to the lung. Only a good powder composition, an accurate measurement device and a properly chosen instrument will accomplish hose objectives. Dry powder inhaler. The gr des are mostly three: NEBULIZER, PMDI and DPI. DPIs give treatment in the form of dry powder to the lung as an alternative to pMDI. API particle size shall be about 1-10 $\mu \mathrm{m}$ in size range, which would also mean that the patient has the same dosage at varying airflow speeds each time. Four forms of formulation methods are formulated to form DPI, for instance: carrier free drug carriers, drug additives, additives from drug carriers. It is important for the internal breathing tool to achieve satisfactory trans ission of respirable medication to the pulmonary system, which basically is grouped into single unit and multi-dosage stores in view of measurements.

Key words: Dry Powder Inhaler, Drug Carrier, Drug Additive, Particle Size, Content Uniformity.

\section{INTRODUCTION:}

The production of formulation involves a number of processes in which an active ingredient in a pharmaceutical product is added. Whereas biological activity is a precondition for an effective dosage type, it is not the only factor. The efficacy of the pharmaceutical system is aided by factors such as safety, processability, distribution and access to the target organ. Optimizing these aspects is a significant production work, and the end result always reflects a balance between realistic (economic/ engineering) and medicinal considerations. Production of formulations is a problem, since pharmacologically active molecules also have weak physicalchemical properties. Indeed, the same molecular features conferred by drug activities (e.g. high receptor affinity) also restrict the medicinal efficacy of a substance and make it impossible or even undesirable for supply..$^{1,2}$

The production of inhalation pharmaceuticals is particularly difficult because it concerns the development of a solution and the selection of a dispersion system for aerosols. ${ }^{3}$ Lung potential is smaller than other transmission sites (e.g., the gastrointestinal tract or blood), which restricts the selection of excipients that can boost distribution results. A more pulmonary supply-unique variable is the patient, in terms of inhalational mode as well as anatomy and physiology of the airway. ${ }^{4}$ There are far other ways of delivering an inhaled aerosol than swallowing a pill. A major variability can 
be achieved in the dosage administered to people or patients.5,6 Therefore it is difficult to guarantee the reproducible therapeutic outcome. Appropriate medications must be administered to the lungs to combat respiratory conditions with inhalation products to produce a medicinal answer. ${ }^{7}$ To achieve optimum effectiveness, effective, reproducible and convenient administration of drugs must be needed. The subsequent discussion discusses the configuration of the Dry Powder Inhaler (DPI) formulations to meet the targets for distribution. The scientifically and therapeutic elements of the device design and selection are discussed thoroughly elsewhere. ${ }^{8-10}$

\section{DRY POWDER INHALERS}

For the last 10-15 years, dry powder inhalers (DPIs) have improved considerably. An inhaler with dry powder (DPI) is a dry-powder product that provides medicine to the lungs. The dry powdering platform contains equipment that generates an aerosol directly from medication powder of 1 to $5 \mu \mathrm{m}$ or blends it with excipients. DPI excipients are used as carrier for the active ingredient in pharmaceuticals (API). Lactose Monohydrate18 is the most widely used carrier. The creation of DPIs was driven by the need to alternatives to pMDI, reducing emissions of chlorofluorocarbons and hydrofluoroalkanes respectively used as propellants of ozone depleting and greenhouse gases. Asthma, bronchitis, emphysema and COPD are widely used for the treatment of respiratory disorders, while DPIs were used also in the treatment of the diabetes mellitus ${ }^{11-12}$.

\section{General Requirements of DPI}

DPIs have to meet the following requirements

Particle Size of API: The inhalable active agent must be. It must be found in particles between 1 to $10 \mu \mathrm{m}$ in order to be able to move through the lung. Micronizing, managed precipitation of the appropriate solvent or spray drying may be accomplished by certain micro-fine particles when methodological conditions are appropriate..$^{13-14}$

Drug content uniformity: It is necessary, in a single-dose system to guarantee that the patient has the same dose per time; the reservoir must therefore release the same quantity of powder and the same volume of medications in the same dose system. ${ }^{15}$

Content uniformity at different airflows: DPI drug distribution relies on the breathing rhythm of the patient. This means that at low breathing and heavy breathing, the dosage needs to be released precisely the same. Consequently, material uniformity is highly essential for a DPI in various airflows. ${ }^{16}$

Stability of powder against humidity and temperature: The administration of DPI drugs is focused on the patient's breathing pattern. This ensures that the dose has to be released almost the same at low breathing and heavy breathing. Content uniformity in multiple airflows is therefore highly important for a DPI. ${ }^{17}$ 


\section{Advantages of Dry Powder Inhaler}

As DPIs has been motivated by the desire for alternatives to pMDIs, so advantages of DPI over pMDI is given as follows;

1. Action and inhalation need little to no synchronisation The prevailing challenge remains the correct use of pMDIs. It was observed, in a significant proportion of patients treated with corticosteroid pMDIs, that inadequate synchronisation of acting and inhaling contributed to reduced asthma regulation. Although DPIs are triggered by the inspiratory airflow of the patient, the movement and inhalation require little to no coordination. This has also contributed to improved lung supply than with equivalent pMDIs. ${ }^{18}$

2. Formulation Consistency Since DPIs are normally formulated as one-phase solid particle blends, the stable formulation is favoured. Dried powders are less efficient, reducing the rate of chemical degradation and the possibility of reaction to touch surfaces. In comparison, pMDI formulations including propellant and solvents can extract organic substances from the components of the system. ${ }^{19}$

3. Propellant-free pMDI includes the propellants of ozone-depleting and greenhouse gases, such as chlorofluorocarbons and hydrofluoroalkans. Since 1 January 1996 it was forbidden to manufacture CFC propellants to avoid ozone depletion. Therefore, pMDI was replaced by nonpropellant-containing DPI. DPIs then make their formulation environmentally safe. ${ }^{19}$
4. The carriage power of heavy drug dosage. DPI can provide a range of doses with one short inhalation from less than $10 \mathrm{mg}$ to more than $20 \mathrm{mg}$.

5. Low oropharyngeal deposition, low retention and low exhaled leakage of the medication, minimal extra pulmonary decline.

6. DPI has less side effects when the majority of the body is not exposed to narcotics, since the drug is deposited in the lung. 4. Less scope for system components to be extracted.

7. Flowability In order to have the right volume of powder for a DPI, this property must be necessary. Owing to a poor flowability of almost all active ingredients, the carrier has to deliver strong flow. ${ }^{20}$

\section{FORMULATION STRATEGIES FOR DPI}

The efficacy of DPI depends primarily upon the powder flow characteristic that is primarily influenced by strong interpartisan forces rendering the bulk powder cohesive agglomerate. The Van der Waals, the electrostatic force, and the capillary force are three types of interparticle forces. When the particles are sufficiently close together $(0.2-1.0 \mathrm{~nm})$ and when the particles are small $(20 \mu \mathrm{m}$ or less) the van der Waals force is recognised. The van der Waals force can dramatically change surface roughness, geometric structure and individual particle deformation. As particles with various working functions come in contact, electrostatic force may emerge from the potential difference. The consequent appeal of Coulomb makes the 
powder adhere. The capillary force is obtained from the condensation of fluid in near contact with particles, which contributes to the creation of liquid bridges between particles. At the cost of the electrostatic force, high capillary pressure decreases with increasing moisture. ${ }^{21}$ To overcome these difficulties different types of formulation strategies for DPI are as follows;

Carrier Free: Effective therapeutic additives, which take the form of a single compound, aggregate or encapsulated particles, are part of carrier free technique. Various processing processes include crystallisation and milling, pulverisation and supercritical fluid. Crystallization and friction were not considered to be ideal for pulmonary drugs because the optimum particle form, the distribution of small particle sizes, low surface energy and amorphous material prevention were not developed. Aerodynamic sample scale less than $5 \mu \mathrm{m}$ must be included in the inhalation medication model. ${ }^{22}$

Drug Carrier: For dry powder inhalers, $1 \mu \mathrm{g}$ to $1 \mathrm{mg}$ of treatment is difficult to dispense in the minor blisters. It's also difficult to inhale powder since the desired particles range from 1 to $5 \mu \mathrm{m}$. The pharmaceutical molecules can also be combined with bigger particles to improve their movement and boost their dosage amount. These carriers can measure between 50 and $100 \mu \mathrm{m}$ in geometry. Cough particles, primed, may be used to help fluidize fine particles as an additional agitator or turbulence promoter. In comparison, it is simpler to dispense with very small amounts of active ingredient in bulked mixture. This technique has a disadvantage: carriers normally deposit into the mouth along with other drug spores, which contribute to less drug in the lungs. The misalignment of medicinal particles from the carriers' surface often contributes to low distribution efficiency. ${ }^{23}$

Drug Additive: Adding fine particles can also increase the fluidization consistency of fine drug powders. The appeal of van der Waals is primarily focused on the particle-particle distance, which greatly decrease adhesive force by increasing the separation distance and therefore enhances liquidation of the fine particles and thus increases the flow characteristics of the substance. Additives such as silica (0.5-3 Wt.\%), alumina (29 $\mathrm{nm}$ ), 200 aerosol (12 $\mathrm{nm}$ ) have been used. The following additives are available: ${ }^{24}$

Drug Carrier Additive: To boost drug delivery, an extra particle type may be applied to the mixture. This additive may be a fine particle such as a fine particle of the same structure as the carrier that may act as a physical separator, or even by occupying energy-intensive spaces such as keys inside the carrier's surface. The most famous example is the use of fine lactose in a carriage method with lactose. An expansion of the lactose fine fraction resulted in greater isolation of medicinal particles from the carrier particle. ${ }^{25}$

\section{CONCLUSION}

Inhalation of drug powder is a rapidly growing field. For a good inhalation product, the system is a significant part. This analysis 
summarises critical categories of dry powder inhaler instruments.

\section{REFERENCES}

[1] Di L, Kerns EH. Profiling drug-like properties in discovery research.Curr Opin Chem Biol 2003;7(3):402-408.

[2] Lipinski CA. Drug-like properties and the causes of poor solubilityand poor permeability. J Pharmacol Toxicol Methods 2000;44(1):235-249.

[3] Lipinski CA, Lombardo F, Dominy BW, Feeney PJ. Experimentaland computational approaches to estimate solubility and permeability in drug discovery and development settings. Adv Drug Deliv Rev 2001;46(1-3):3-26.

[4] Timsina MP, Martin GP, Marriott C, et al. Drug-delivery to the respiratory-tract using dry powder inhalers. Int J Pharm 1994;101(1):1-13.

[5] Aswania 0, Ritson S, Iqbal SM, Bhatt J, Rigby AS, Everard ML. Intra-subject variability in lung dose in healthy volunteers using five conventional portable inhalers. J Aerosol Med 2004;17(3):231-238.

[6] Smyth HD, Hickey AJ. Carriers in drug powder delivery: implications for inhalation system design. Am J Drug Deliv 2005;3(2)117-132.

[7] Dolovich MB, Ahrens RC, Hess DR, Anderson P, Dhand R, Rau JL,et al. Device selection and outcomes of aerosol therapy: evidencebased guidelines: American College of Chest Physicians/American College of Asthma, Allergy, and Immunology. Chest 2005;127(1):335-371.

[8] O'Connor BJ. The ideal inhaler: design and characteristics to improve out comes. Respir Med 2004;98 Suppl A:S10-S16.
[9] Newman SP, Clarke SW. Bronchodilator delivery from Gentlehaler, a new lowvelocity pressurized aerosol inhaler. Chest 1993;103(5):1442-1446.

[10] Ganderton D. General factors influencing drug delivery to the lung. Respir Med 1997;91 Suppl A:13-16.

[11] Newman SP, Newhouse MT. Effect of addon devices for aerosol drug delivery: deposition studies and clinical aspects. J Aerosol Med 1996;9(1):55-70.

[12] Giraud V, Roche N. Misuse of corticosteroid metered-dose inhaler is associated with decreased asthma stability. Eur Respir J 2002;19(2):246251.

[13] Borgstrom L, Derom E, Stahl E, WahlinBoll E, Pauwels R. The inhalation device influences lung deposition and bronchodilating effect of terbutaline. Am J Respir Crit Care Med 1996;153(5):16361640.

[14] Ashurst II, Malton A, Prime D, Sumby B. Latest advances in the development of dry powder inhalers. Pharm Sci Technol 2000;3(7):246-256.

[15] Norwood DL, Prime D, Downey BP, Creasey J, Sethi SK, Haywood P. Analysis of polycyclic aromatic hydrocarbons in metered dose inhaler drug formulations by isotope dilution gas chromatography/mass spectrometry. J Pharm Biomed Anal 1995;13(3):293-304.

[16] Dalby R, Suman J. Inhalation therapy: technological milestones in asthma treatment. Adv Drug Deliv Rev 2003;55(7):779-791.

[17] Newman SP, Busse WW. Evolution of dry powder inhaler design, formulation, and performance. Respir Med 2002;96(5):293-304. 
[18] Dunbar CA, Hickey AJ, Holzner P. Dispersion and characterization of pharmaceutical dry powder aerosols. Kona 1998;16:7-44.

[19] Smith IJ, Parry-Billings M. The inhalers of the future? A review of dry powder devices on the market today. Pulm Pharmacol Ther2003;16(2):79-95.
[20] Newman S, Hollingworth A, Clark A. Effect of different modes of inhalation on drug delivery from a dry powder inhaler. Int J Pharma 1994;102(1):127-132.

[21] Cegla UH. Pressure and inspiratory flow characteristics of dry powder inhalers. Respir Med 2004;98 Suppl A:S22-S28.

How to cite this article:

Prasanna Kumar K et al A Review on Dry Powder Inhalers. Int. J. Adv. Pharm. Biotech., 2015; 1(4): 1-6. 